\title{
Correction to: An evaluation of the structures of cellulose generated by the CHARMM force field: comparisons to in planta cellulose
}

\author{
Daniel P. Oehme $\cdot$ Hui Yang $\cdot$ James D. Kubicki
}

Published online: 14 May 2018

(C) Springer Science+Business Media B.V., part of Springer Nature 2018

\section{Correction to: Cellulose \\ https://doi.org/10.1007/s10570-018-1793-4}

In the original publication of the article, the authors have not properly acknowledged the funding sources in the acknowledgments. Hence, the updated acknowledgment is provided below.

Acknowledgments The work by H.Y. and J.D.K., and analysis and writing of the manuscript by D.P.O. were supported by the Center for Lignocellulose Structure and Formation, an Energy Frontier Research Center funded by the US Department of Energy, Office of Science, Basic Energy Sciences under Award \# DE-SC0001090. The performance of
MD simulations by D.P.O. while at the University of Melbourne was funded by a grant from the Australia Research Council (ARC) to the ARC Centre of Excellence in Plant Cell Walls [CE110001007], and the Victorian Life Sciences Computation Initiative (VLSCI) grant number "VR0319" on its Peak Computing Facility at the University of Melbourne, an initiative of the Victorian State Government. Parts of this work were completed while D.P.O. was at IBM ResearchAustralia. Portions of this research were conducted with Advanced Cyber Infrastructure computational resources provided by the Institute for Cyber Science at The Pennsylvania State University (http://ics.psu.edu). We also thank Prof. Mei Hong for the experimental triple mutant intact Arabidopsis spectra.
The original article can be found online at https:// doi.org/10.1007/s10570-018-1793-4.

D. P. Oehme · J. D. Kubicki

Department of Geological Sciences, University of Texas at El Paso, El Paso, TX, USA

D. P. Oehme $(\square)$

ARC Centre of Excellence in Plant Cell Walls, School of BioSciences, The University of Melbourne, Parkville,

VIC, Australia

e-mail: dpoehme@utep.edu

H. Yang

Department of Biology, Pennsylvania State University, University Park, PA, USA 